\title{
LIMITING BEHAVIOUR OF THE MEAN RESIDUAL LIFE
}

\author{
David M. Bradley and Ramesh C. Gupta \\ Department of Mathematics and Statistics, University of Maine, Orono, ME \\ 04469-5752, U.S.A. \\ e-mail: bradley@math.umaine.edu,rcgupta@maine.maine.edu
}

Key words and phrases: Mean residual life, limiting behaviour, asymptotic expansion, failure rate, hazard function.

\begin{abstract}
In survival or reliability studies, the mean residual life or life expectancy is an important characteristic of the model. Here, we study the limiting behaviour of the mean residual life, and derive an asymptotic expansion which can be used to obtain a good approximation for large values of the time variable. The asymptotic expansion is valid for a quite general class of failure rate distributions - perhaps the largest class that can be expected given that the terms depend only on the failure rate and its derivatives.
\end{abstract}

Date: Submitted March 7, 2001; revised May 29, 2002; accepted June 12, 2002. 


\section{INTRODUCTION AND BACKGROUND}

In life testing situations, the expected additional lifetime given that a component has survived until time $t$ is a function of $t$, called the mean residual life. More specifically, if the random variable $X$ represents the life of a component, then the mean residual life is given by $m(t)=E(X-t \mid X>t)$. It is well known that the mean residual life is related to the survival (reliability) function $\bar{F}$ by

$$
m(t)=\frac{1}{\bar{F}(t)} \int_{t}^{\infty} \bar{F}(u) d u
$$

and to the failure rate (hazard function) $r=-\bar{F}^{\prime} / \bar{F}$ by

$$
m(t)=\int_{t}^{\infty} \exp \left\{-\int_{t}^{u} r(x) d x\right\} d u=e^{R(t)} \int_{t}^{\infty} e^{-R(u)} d u
$$

where

$$
R(t)=\int_{0}^{t} r(x) d x=-\log \bar{F}(t)
$$

is the integrated failure rate (cumulative hazard function). We also have

$$
m^{\prime}(t)=r(t) m(t)-1
$$

(see Calabria and Pulcini (1987), for example.)

The mean residual life has been employed in life length studies by various authors, e.g. Hollander and Proschan (1975), Bryson and Siddiqui (1969), and Muth (1977). Muth (1977) observed that the failure rate takes only the instantaneous present into account, whereas the mean residual life takes the complete future into account. Meilijson (1972) 
has studied certain limiting properties of the mean residual life. A smooth estimator of the mean residual life is given by Chaubey and Sen (1999).

In this paper, we undertake a detailed study of the limiting behaviour of the mean residual life (\$2), and derive an asymptotic expansion (\$3) which can be used to obtain good approximations for large values of the time variable. The asymptotic expansion is valid for a quite general class of failure rate distributions - perhaps the largest class that can be expected given that the terms depend only on the failure rate and its derivatives.

For the family of age smooth distributions, Rojo (1996) has established, for large values of the time variable, a relationship between the mean residual life and the failure rate in terms of the index $\rho$ of regular variation. Calabria and Pulcini (1987) noted a relationship between the limiting behaviour of the mean residual life and the failure rate. This relationship was developed further by Chaubey and Sen (1999) for the class of distributions having nondecreasing failure rate. Our approach provides a considerable improvement on their approximation, and moreover does not require that the failure rate be nondecreasing.

\section{Limiting Behaviour}

By applying L'Hôpital's rule to (1.1), Calabria and Pulcini (1987) derived the relationship

$$
\lim _{t \rightarrow \infty} m(t)=\lim _{t \rightarrow \infty} \frac{1}{r(t)}
$$


provided the latter limit exists and is finite. They then used (1.4) to conclude that $\lim _{t \rightarrow \infty} m^{\prime}(t)=0$, or equivalently, that

$$
\lim _{t \rightarrow \infty} r(t) m(t)=1
$$

Unfortunately, one cannot infer (2.2) from (2.1) unless one also assumes that $\lim _{t \rightarrow \infty} r(t)$ is finite and strictly positive. For a counterexample, fix positive constants $a$ and $b$ and consider the linear mean residual life $m(t)=a+b t$ with corresponding failure rate $r(t)=$ $(1+b) /(a+b t)$. The class of distributions with linear mean residual life has been studied by Hall and Wellner (1981) and Oakes and Dasu (1990). Counterexamples that satisfy $\lim _{t \rightarrow \infty} r(t)=\infty$ also exist, but as these tend to be somewhat more complicated, further discussion is deferred to the end of this section. A more detailed study of the limiting behaviour of the mean residual life follows.

Theorem 1. Let $r$ denote the failure rate, and let $m$ denote the corresponding mean residual life. For $t>0$ define $\beta(t)=\operatorname{ess} \sup \{r(x): x>t\}$ and $\alpha(t)=\operatorname{ess} \inf \{r(x): x>$ $t\}$. Then

$$
\lim _{t \rightarrow \infty} \frac{1}{\beta(t)} \leq \liminf _{t \rightarrow \infty} m(t) \leq \limsup _{t \rightarrow \infty} m(t) \leq \lim _{t \rightarrow \infty} \frac{1}{\alpha(t)}
$$

Proof. Since $\alpha$ (respectively, $\beta$ ) is clearly nondecreasing (nonincreasing),

$$
m(t)=\int_{0}^{\infty} \exp \left\{-\int_{t}^{x+t} r(y) d y\right\} d x
$$

implies

$$
\int_{0}^{\infty} e^{-x \beta(t)} d x \leq m(t) \leq \int_{0}^{\infty} e^{-x \alpha(t)} d x
$$


Corollary 1. The failure rate $r$ and the mean residual life $m$ have limiting behaviour related by

$$
\varlimsup_{t \rightarrow \infty} \frac{1}{\limsup _{t \rightarrow \infty} r(t)} \leq \liminf _{t \rightarrow \infty} m(t) \leq \limsup _{t \rightarrow \infty} m(t) \leq \frac{1}{\liminf _{t \rightarrow \infty} r(t)}
$$

Note that Corollary 1 implies (2.1) without the assumption that $\lim _{t \rightarrow \infty} 1 / r(t)$ exists; for example, if $\limsup _{t \rightarrow \infty} r(t)=0$, then $\lim _{t \rightarrow \infty} m(t)=\infty$. Corollary 1 also implies that if $\lim _{t \rightarrow \infty} r(t)$ exists (finite) and is strictly positive, then (2.2) holds.

The limiting reciprocal relationship (2.2) between the failure rate and the mean residual life may be interpreted as an approximation or asymptotic formula $m \sim s$, where $s=1 / r$. By imposing suitable conditions on $s$ and its derivatives, it is possible to refine this approximation by introducing additional terms into the asymptotic formula. We shall carry out this programme in \$3, Even in cases where the reciprocal relationship (2.2) fails, one can sometimes obtain reasonably precise information about the limiting behaviour of the product of the failure rate and the mean residual life by studying the limiting behaviour of $s$ and its derivatives. By putting $E=[0, \infty)$ in Corollary 2 below, it can be seen that for continuously differentiable $s$, if $\lim _{t \rightarrow \infty} s^{\prime}(t)=0$, then (2.2) holds. However, it may happen that $\lim _{t \rightarrow \infty} s^{\prime}(t)$ does not exist. It turns out that if $\left|s^{\prime}(t)\right|$ is not ultimately "too big too often," one can still say a good deal about the product $r(t) m(t)$ when $t$ is large: see Theorem 4 and Corollary 2 below. There are also failure rates for which nonzero 
values of $\lim _{t \rightarrow \infty} s^{\prime}(t)$ are possible. In such cases, the reciprocal relationship (2.2) may fail. Nevertheless, we have the following result.

Theorem 2. Denote the failure rate and its reciprocal by $r$ and $s=1 / r$, respectively, and let $m$ denote the corresponding mean residual life. Let $R$ denote the integrated failure rate (1.3). Suppose that

$$
\lim _{t \rightarrow \infty} s(t) \exp (-R(t))=0
$$

and that

$$
\lim _{t \rightarrow \infty}\left(1-s^{\prime}(t)\right)^{-1} \text { exists (finite) }
$$

Then

$$
\lim _{t \rightarrow \infty} r(t) m(t)=\lim _{t \rightarrow \infty}\left(1-s^{\prime}(t)\right)^{-1}
$$

Proof. Apply L'Hôpital's rule to

$$
r(t) m(t)=\int_{t}^{\infty} e^{-R(x)} d x /\left(s(t) e^{-R(t)}\right)
$$

When applying Theorem 2 in practice, it may often be easier to verify conditions that imply the hypothesis (2.3), as opposed to verifying (2.3) directly. We give an example of such a condition below. 
Theorem 3. Denote the reciprocal of the failure rate by s, and let $R$ denote the integrated failure rate (1.3). Suppose that $\lim _{t \rightarrow \infty} \operatorname{ess} \sup \left\{s^{\prime}(x): x>t\right\}<1$ and that $s(t)$ is finite for all sufficiently large values of $t$. Then (2.3) holds.

Proof. There exist numbers $L<1$ and $t_{0} \geq 0$ such that for all $t \geq t_{0}, s(t)$ is finite and ess $\sup \left\{s^{\prime}(x): x>t\right\}<L$. For $t \geq t_{0}$, we have

$$
\begin{aligned}
s(t) \exp (-R(t)) & =\exp \left\{\log s\left(t_{0}\right)+\int_{t_{0}}^{t} \frac{s^{\prime}(x)}{s(x)} d x-\int_{0}^{t} r(x) d x\right\} \\
& =s\left(t_{0}\right) \exp \left\{\int_{t_{0}}^{t}\left(s^{\prime}(x)-1\right) r(x) d x-\int_{0}^{t_{0}} r(x) d x\right\} \\
& \leq s\left(t_{0}\right) \exp \left\{\left(\underset{x>t_{0}}{\operatorname{ess} \sup } s^{\prime}(x)-1\right) \int_{t_{0}}^{t} r(x) d x-R\left(t_{0}\right)\right\} \\
& \leq s\left(t_{0}\right) \exp \left\{(L-1)\left(R(t)-R\left(t_{0}\right)\right)-R\left(t_{0}\right)\right\} \\
& =s\left(t_{0}\right) e^{-L R\left(t_{0}\right)}(\bar{F}(t))^{1-L} .
\end{aligned}
$$

Since $\lim _{t \rightarrow \infty} \bar{F}(t)=0$ and $L<1$, (2.3) follows.

A condition that implies the hypothesis $\lim _{t \rightarrow \infty} \operatorname{ess} \sup \left\{s^{\prime}(x): x>t\right\}<1$ of Theorem [3] and is typically even easier to verify is $\lim _{\sup _{t \rightarrow \infty}} s^{\prime}(t)<1$. Thus, Theorems 2 and 3 can be readily applied to a wide variety of situations in which the long term behaviour of the rate of change of the reciprocal of the failure rate is known. A very simple yet illustrative example is provided by the distribution with fractional linear failure rate $r(t)=1 /(c+d t)$. Here $s^{\prime}$ has constant value $d$, and thus if $d<1$, then Theorems 2 
and 3 imply that $\lim _{t \rightarrow \infty} r(t) m(t)=1 /(1-d)$. Of course, in this case it is easy to verify this fact directly, as the mean residual life function is linear: $m(t)=(c+d t) /(1-d)$. However, Theorems 2 and 3 are equally applicable to cases in which it may be difficult or impossible to determine the long term behaviour of the mean residual life directly.

The issue of practical usefulness apart, there are compelling theoretical reasons which point up the significance of Theorem 2 as well. Recall the observation that (2.2) may be interpreted as an approximation or asymptotic formula $m \sim s$. Viewing the conclusion of Theorem 2 in the same light yields

$$
m \sim \frac{s}{1-s^{\prime}}=s\left(1+s^{\prime}+\left(s^{\prime}\right)^{2}+\left(s^{\prime}\right)^{3}+\cdots\right), \quad\left|s^{\prime}\right|<1,
$$

which agrees with the first three terms of the asymptotic expansion (3.6)). Thus, in some sense, Theorem 2 represents a strengthening of Calabria and Pulcini's first order result (2.2) - subject to the appropriate conditions - to third order.

We next address the problem of what happens when $s^{\prime}$ exists but $\lim _{t \rightarrow \infty} s^{\prime}(t)$ does not. As might be expected, in general some information about the limiting behaviour of the product $r(t) m(t)$ is lost. Nevertheless, in many cases one can at least bound $r m$ in terms of the essential supremum of $s^{\prime}$. We'll see as a result that even if $\lim _{\sup } \operatorname{su}_{t \rightarrow \infty}\left|s^{\prime}(t)\right|>0$, as long as $\left|s^{\prime}(t)\right|$ is not ultimately "too big too often" then (2.2) holds.

Theorem 4. Let $r$ denote the failure rate, and let $m$ denote the corresponding mean residual life. Let $s=1 / r$, and put $\lambda(t):=\operatorname{ess} \sup \left\{\left|s^{\prime}(x)\right|: x>t\right\}$. Suppose that $r(t)$ is positive and continuously differentiable for all sufficiently large values of $t$, and that 
$\lim _{t \rightarrow \infty} \lambda(t)<1$. Then for all sufficiently large values of $t, 1 /(1+\lambda(t)) \leq r(t) m(t) \leq$ $1 /(1-\lambda(t))$.

Proof. By Theorem 3, condition (2.3) holds. Thus, the hypotheses permit us to integrate by parts and discard the limit at the upper range of integration in the integrated term. We have for all sufficiently large values of $t$,

$$
r(t) m(t)=r(t) e^{R(t)} \int_{t}^{\infty} r(x) e^{-R(x)} s(x) d x=1+r(t) e^{R(t)} \int_{t}^{\infty} e^{-R(x)} s^{\prime}(x) d x .
$$

But

$$
\left|\int_{t}^{\infty} e^{-R(x)} s^{\prime}(x) d x\right| \leq \underset{x>t}{\operatorname{ess} \sup }\left|s^{\prime}(x)\right| \int_{t}^{\infty} e^{-R(x)} d x
$$

Thus, for all sufficiently large values of $t$,

$$
1-\lambda(t) r(t) m(t) \leq r(t) m(t) \leq 1+\lambda(t) r(t) m(t) .
$$

It follows that the reciprocal relationship (2.2) may hold even if $\lim _{\sup } \rightarrow \infty\left|s^{\prime}(t)\right|>0$, as long as $\lim _{n \rightarrow \infty} s^{\prime}\left(t_{n}\right)=0$ for "most" sequences $t_{1}<t_{2}<\cdots \rightarrow \infty$. Here, "most" is in the sense of Lebesgue measure. The following result makes this observation more precise.

Corollary 2. Let $s$ denote the reciprocal of the failure rate. Suppose that $s(t)$ is finite and continuously differentiable for all sufficiently large values of $t$. Suppose further that there exists a subset $E$ of the interval $[0, \infty)$ whose complement in $[0, \infty)$ is of Lebesgue measure 
zero, and such that for every sequence $t_{1}, t_{2}, \ldots$ of elements of $E$ with $\lim _{n \rightarrow \infty} t_{n}=\infty$ we have $\lim _{n \rightarrow \infty} s^{\prime}\left(t_{n}\right)=0$. Then (2.2) holds.

Proof. As customary, denote the indicator function of a set $A$ by $\chi_{A}$. Suppose that $E \subseteq[0, \infty)$ satisfies the hypotheses of the Corollary. Then the complement of $E$ in $[0, \infty)$ has Lebesgue measure zero, and $\lim _{x \rightarrow \infty} s^{\prime}(x) \chi_{E}(x)=0$. Therefore, if $\varepsilon>0$ is given, there exists a suitably large value of $t$ such that $\left|s^{\prime}(x) \chi_{[t, \infty)}(x)\right|<\varepsilon$ for all $x \in E$. By definition of the essential supremum, this implies (in the notation of Theorem 4) that $\lambda(t)=\operatorname{ess} \sup \left\{\left|s^{\prime}(x)\right|: x>t\right\}<\varepsilon$. As $\lambda$ is a nonincreasing function and $\varepsilon>0$ is arbitrary, it follows that $\lim _{t \rightarrow \infty} \lambda(t)=0$.

We conclude this section with an example of a distribution in which $\lim _{t \rightarrow \infty} r(t)=\infty$, but $\lim _{t \rightarrow \infty} r(t) m(t) \neq 1$. Let $a, b, c, d$ be positive constants satisfying $a>b, d>2 b$, and $c^{2}>(a+b) d$. Consider the mean residual life defined by

$$
m(t)=\frac{a+b \sin \left(t^{2}\right)}{c+d t}, \quad t \geq 0 .
$$

We have

$$
m^{\prime}(t)=\frac{2 b t \cos \left(t^{2}\right)}{c+d t}-\frac{\left(a+b \sin \left(t^{2}\right)\right) d}{(c+d t)^{2}}
$$

Thus,

$$
\liminf _{t \rightarrow \infty} m^{\prime}(t)=-2 b / d, \quad \quad \limsup _{t \rightarrow \infty} m^{\prime}(t)=2 b / d,
$$

and hence

$$
\liminf _{t \rightarrow \infty} r(t) m(t)=1+\liminf _{t \rightarrow \infty} m^{\prime}(t)=1-2 b / d<1
$$


whereas

$$
\limsup _{t \rightarrow \infty} r(t) m(t)=1+\limsup _{t \rightarrow \infty} m^{\prime}(t)=1+2 b / d>1 .
$$

Since

$$
\begin{aligned}
(c+d t)^{2}\left(1+m^{\prime}(t)\right) & =(c+d t)^{2}+(c+d t) 2 b t \cos \left(t^{2}\right)-\left(a+b \sin \left(t^{2}\right)\right) d \\
& \geq c^{2}+2 c d t+d^{2} t^{2}-2 b c t-2 b d t^{2}-(a+b) d \\
& =(d-2 b) d t^{2}+(d-b) 2 c t+c^{2}-(a+b) d
\end{aligned}
$$

is clearly positive for $t \geq 0$, it follows that $r(t)=\left(1+m^{\prime}(t)\right) / m(t)>0$ for $t \geq 0$. Furthermore,

$$
r(t)=\frac{c+\left(d+2 b \cos \left(t^{2}\right)\right) t}{a+b \sin \left(t^{2}\right)}-\frac{d}{c+d t}
$$

implies $\lim _{t \rightarrow \infty} r(t)=\infty$.

\section{Asymptotic Expansion}

Under certain conditions, the mean residual life has an asymptotic expansion in terms of the failure rate and its derivatives. An initial attempt in this direction was made by Chaubey and Sen (1999) for the class of distributions having nondecreasing failure rate. However, it is easy to envision situations where, say with regular maintenance, even an ultimately nondecreasing failure rate may be an inappropriate model. Therefore, we provide an alternative approach that requires no monotonicity assumptions on the failure rate. 
We take Chaubey and Sen's asymptotic formula

$$
m(t)=\frac{1}{r(t)}-\frac{r^{\prime}(t)}{(r(t))^{3}}+O\left(\frac{r^{\prime \prime}(t)}{(r(t))^{4}}\right), \quad t \rightarrow \infty
$$

(with error term corrected), as a point of departure. Unfortunately, their derivation is not rigorous, and as a result, certain growth conditions on $r$ and its derivatives are omitted. Nevertheless, their approach can, in principle, be made to work, and thus one can show that under suitable conditions on the failure rate there is an asymptotic series development to arbitrary order that begins

$$
m \sim r^{-1}-r^{\prime} r^{-3}-r^{\prime \prime} r^{-4}+\left(3\left(r^{\prime}\right)^{2}-r^{\prime \prime \prime}\right) r^{-5}+\left(10 r^{\prime} r^{\prime \prime}-r^{\prime \prime \prime \prime}\right) r^{-6}+\cdots, \quad t \rightarrow \infty
$$

Here, we have made the abbreviations $m=m(t), r^{-1}=1 / r(t)$, etc. More explicitly, for each positive integer $n$, we have

$$
m \sim \sum_{k=0}^{n-1} c_{k} r^{-k-1}+(\text { additional terms })
$$

where as $t \rightarrow \infty$, the additional terms tend to zero more rapidly than $c_{k} r^{-k-1}$ for $1 \leq$ $k \leq n-1$. Here, $c_{0}=1, c_{1}=0, c_{2}=-r^{\prime}, c_{3}=-r^{\prime \prime}, c_{4}=3\left(r^{\prime}\right)^{2}-r^{\prime \prime \prime}$, and in general, $c_{k}$ is a polynomial in $r^{\prime}, r^{\prime \prime}, \ldots, r^{(k-1)}$ for $k \geq 2$, the explicit form of which is given by

$$
c_{k}=c_{k}(t)=k ! \sum_{p=0}^{\lfloor k / 2\rfloor}(-1)^{p} \sum \prod_{j \geq 1} \frac{1}{\alpha_{j} !}\left(\frac{r^{(j)}(t)}{(j+1) !}\right)^{\alpha_{j}}
$$

where the inner sum is over all nonnegative integers $\alpha_{1}, \alpha_{2}, \ldots$ such that $\sum_{j \geq 1} \alpha_{j}=p$ and $\sum_{j \geq 1}(j+1) \alpha_{j}=k$ 
Rather than attempt a rigorous proof of (3.1) and (3.2) along these lines, we instead develop an alternative, operator-theoretic approach that appears to be simpler, yet more powerful. By way of motivation, observe that since we are interested in the situation when (2.2) holds, it makes sense to let $s=1 / r$ and rewrite the differential equation (1.4) in the form $(1-s D) m=s$, where $D$ denotes the derivative operator. Abbreviating $s D$ by $\Theta$, we might hope to find a meaningful way to define the inverse operator $(1-\Theta)^{-1}$ in such a way that given suitable growth conditions on $s$ and its derivatives, we have a legitimate asymptotic expansion

$$
m=(1-\Theta)^{-1} s \sim s+\Theta(s)+\Theta^{2}(s)+\Theta^{3}(s)+\cdots
$$

That this is indeed the case is the main content of Theorem 5 , in which an explicit formula is also given for $\Theta^{k}(s)$, the $k$ th term of the expansion. A significant step in this direction is provided by the following result.

Lemma 1. Let $s$ denote the reciprocal of the failure rate, and suppose that for some nonnegative integer $n, s$ is $n+1$ times continuously differentiable on the positive real line. As usual, let $R$ denote the integrated failure rate (1.3), and let $D$ denote the derivative operator. For positive integers $k$, let $\Theta^{k}:=(s D)^{k}$ and for convenience, let $\Theta^{0}:=s$. If in addition, we have $\lim _{x \rightarrow \infty} \exp (-R(x)) \Theta^{k}(s(x))=0$ for each $k=0,1, \ldots, n$, then the mean residual life can be expressed in the form

$$
m(t)=s(t)+\sum_{k=1}^{n} \Theta^{k}(s(t))+e^{R(t)} \int_{t}^{\infty} r(x) e^{-R(x)} \Theta^{n+1}(s(x)) d x, \quad t \geq 0 .
$$


Proof. The conditions on $s$ permit us to integrate by parts $n+1$ times and discard the limits at the upper range of integration. Thus, integrating by parts once, we have

$$
m(t)=e^{R(t)} \int_{t}^{\infty} r(x) e^{-R(x)} s(x) d x=s(t)+e^{R(t)} \int_{t}^{\infty} r(x) e^{-R(x)} \Theta(s(x)) d x .
$$

It is now evident that the claimed formula (3.3) can be proved using mathematical induction and repeatedly integrating by parts.

Alternatively, note that $(1-s(t) D) \exp (R(t)) \int_{t}^{\infty} r(x) \exp (-R(x)) h(x) d x=h(t)$ for all locally integrable functions $h$ for which the integral converges, and so we may define

$$
(1-\Theta)^{-1} h(t)=e^{R(t)} \int_{t}^{\infty} r(x) e^{-R(x)} h(x) d x
$$

for all such functions $h$. If we now take $h=s$ and then $h=\Theta^{n+1} s$, then in light of the identity

$$
(1-\Theta)^{-1}=1+\Theta+\Theta^{2}+\cdots+\Theta^{n}+(1-\Theta)^{-1} \Theta^{n+1}
$$

we have

$$
\begin{aligned}
m(t) & =e^{R(t)} \int_{t}^{\infty} r(x) e^{-R(x)} s(x) d x \\
& =(1-\Theta)^{-1} s(t) \\
& =s(t)+\sum_{k=1}^{n} \Theta^{k}(s(t))+(1-\Theta)^{-1} \Theta^{n+1}(s(t)) \\
& =s(t)+\sum_{k=1}^{n} \Theta^{k}(s(t))+e^{R(t)} \int_{t}^{\infty} r(x) e^{-R(x)} \Theta^{n+1}(s(x)) d x,
\end{aligned}
$$

as claimed. 
Theorem 5. Let s denote the reciprocal of the failure rate. Suppose that for some nonnegative integer $n, s$ is $n+1$ times continuously differentiable on the positive real line, and that $\lim _{t \rightarrow \infty} s(t) \exp (-R(t))=0$, where as usual $R$ denotes the integrated failure rate (1.3). Let $D$ and $\Theta$ be as in Lemma 1. Suppose that for each $k=0,1, \ldots, n$ we have $s(t) s^{(k+1)}(t)=o\left(s^{(k)}(t)\right)$ as $t \rightarrow \infty$. Then $\Theta^{k+1}(s)=o\left(\Theta^{k}(s)\right)$ for all $0 \leq k \leq n$, and we have the asymptotic expansion

$$
m(t)=s(t)+\sum_{k=1}^{n} \Theta^{k}(s(t))+o\left(\Theta^{n}(s(t))\right), \quad t \rightarrow \infty
$$

for the mean residual life. The terms $\Theta^{k}(s)$ may be expressed in terms of $s$ and its derivatives by means of

$$
\Theta^{k}(s)=s \sum_{j_{1}, \ldots, j_{k} \geq 0} d\left(j_{1}, \ldots, j_{k}\right) \prod_{p=1}^{k} s^{\left(j_{p}\right)}
$$

where the sum extends over all nonnegative integers $j_{p}(1 \leq p \leq k)$ and the coefficients $d\left(j_{1}, \ldots, j_{k}\right)$ are generated by the polynomial equation

$$
\sum_{j_{1}, \ldots, j_{k} \geq 0} d\left(j_{1}, \ldots, j_{k}\right) \prod_{p=1}^{k} x_{p}^{j_{p}}=\prod_{p=1}^{k} \sum_{j=1}^{p} x_{j}
$$

in the indeterminates $x_{1}, \ldots, x_{k}$.

Proof. The stated formula for $\Theta^{k}(s)$ is a special case of a more general result. See Snyder (1982). We next show that, under the hypotheses on $\left.s, \Theta^{k+1}(s)=o\left(\Theta^{k}(s)\right)\right)$ for 
$0 \leq k \leq n$. Since $\Theta^{k+1}(s)=s D \Theta^{k}(s)$, we have

$$
\begin{aligned}
\Theta^{k+1}(s) & =s D s \sum_{j_{1}, \ldots, j_{k} \geq 0} d\left(j_{1}, \ldots, j_{k}\right) \prod_{p=1}^{k}\left(D^{j_{p}} s\right) \\
& =s^{\prime} \Theta^{k}(s)+s \sum_{j_{1}, \ldots, j_{k} \geq 0} d\left(j_{1}, \ldots, j_{k}\right) s D \prod_{p=1}^{k}\left(D^{j_{p}} s\right) \\
& =s^{\prime} \Theta^{k}(s)+s \sum_{j_{1}, \ldots, j_{k} \geq 0} d\left(j_{1}, \ldots, j_{k}\right) \sum_{q=1}^{k}\left(s D^{j_{q}+1} s\right) \prod_{\substack{p=1 \\
p \neq q}}^{k}\left(D^{j_{p}} s\right) .
\end{aligned}
$$

Note that the generating function (3.5) implies that $j_{1}+\cdots+j_{k}=k$ above. In particular, since $0 \leq k \leq n$, each $j_{q} \leq n$, and so each $s D^{j_{q}+1} s=o\left(D^{j_{q}} s\right)$. Also, $s^{\prime}=o(1)$ because $s s^{\prime}=s D s=o(s)$. Therefore, we have

$$
\begin{aligned}
\Theta^{k+1}(s) & =o\left(\Theta^{k}(s)\right)+o\left(\sum_{q=1}^{k} s \sum_{j_{1}, \ldots, j_{k} \geq 0} d\left(j_{1}, \ldots, j_{k}\right) \prod_{p=1}^{k} D^{j_{p}} s\right) \\
& =o\left(\Theta^{k}(s)\right)+o\left(k \Theta^{k}(s)\right) \\
& =o\left(\Theta^{k}(s)\right) .
\end{aligned}
$$

Next, observe that since $\lim _{t \rightarrow \infty} s(t) \exp (-R(t))=0$ and $\Theta^{k+1}(s)=o\left(\Theta^{k}(s)\right)$ for all $k=0,1, \ldots, n$, we have $\lim _{t \rightarrow \infty} \exp (-R(t)) \Theta^{k}(s(t))=0$ for all $k=0,1, \ldots, n$. Therefore, in light of Lemma 11, it remains only to show that

$$
m(t)-\sum_{k=0}^{n} \Theta^{k}(s(t))=e^{R(t)} \int_{t}^{\infty} r(x) e^{-R(x)} \Theta^{n+1}(s(x)) d x=o\left(\Theta^{n}(s(t))\right), \quad t \rightarrow \infty .
$$


Since $\Theta^{n+1}(s)=o\left(\Theta^{n}(s)\right)$, Lemma 1 gives

$$
\begin{aligned}
m(t)-\sum_{k=0}^{n} \Theta^{k}(s(t)) & =e^{R(t)} \int_{t}^{\infty} r(x) e^{-R(x)} \Theta^{n+1}(s(x)) d x \\
& =o\left(e^{R(t)} \int_{t}^{\infty} r(x) e^{-R(x)} \Theta^{n}(s(x)) d x\right) \\
& =o\left(m(t)-\sum_{k=0}^{n-1} \Theta^{k}(s(t))\right) .
\end{aligned}
$$

Therefore,

$$
(1-o(1))\left(m(t)-\sum_{k=0}^{n-1} \Theta^{k}(s(t))\right)=\Theta^{n}(s(t))
$$

from which it follows that

$$
m(t)-\sum_{k=0}^{n-1} \Theta^{k}(s(t))=\Theta^{n}(s(t))(1+o(1))
$$

or equivalently,

$$
m(t)-\sum_{k=0}^{n} \Theta^{k}(s(t))=o\left(\Theta^{n}(s(t))\right)
$$

as claimed.

We have $\Theta^{0}(s)=s, \Theta(s)=s s^{\prime}, \Theta^{2}(s)=s\left(s^{\prime}\right)^{2}+s^{2} s^{\prime \prime}, \Theta^{3}(s)=s\left(s^{\prime}\right)^{3}+4 s^{2} s^{\prime} s^{\prime \prime}+s^{3} s^{\prime \prime \prime}$, and $\Theta^{4}(s)=s\left(s^{\prime}\right)^{4}+11 s^{2}\left(s^{\prime}\right)^{2} s^{\prime \prime}+4 s^{3}\left(s^{\prime \prime}\right)^{2}+7 s^{3} s^{\prime} s^{\prime \prime \prime}+s^{4} s^{\prime \prime \prime \prime}$. Thus, under the conditions of Theorem [5, our asymptotic expansion begins

$$
m \sim s+s s^{\prime}+s\left(s^{\prime}\right)^{2}+s^{2} s^{\prime \prime}+s\left(s^{\prime}\right)^{3}+4 s^{2} s^{\prime} s^{\prime \prime}+s^{3} s^{\prime \prime \prime}+\cdots, \quad t \rightarrow \infty .
$$


Acknowledgement. The authors are grateful to the anonymous referees for a careful reading of the original manuscript. Their suggestions contributed to improvements in the exposition.

\section{REFERENCES}

[1] Bryson, C. and Siddiqui, M. M. (1969). "Some criteria for aging." Journal of the American Statistical Association, 64, 1472-1483.

[2] Calabria, R. and Pulcini, G. (1987). "On the asyptotic behaviour of the mean residual life function." Reliability Engineering, 19, 165-170.

[3] Chaubey, Y. P. and Sen, P. K. (1999). "On smooth estimation of mean residual life." Journal of Statistical Planning and Inference, 75, 223-236.

[4] Hall, W. J. and Wellner, J. A. (1981). "Mean residual life." Proceedings of the International Symposium on Statistics and Related Topics, (eds. M. Csörgö, D. A. Dawson, J. N. K. Rao, and A. K. M. E. Saleh), 169-184.

[5] Hollander, W. and Proschan, F. (1975). "Tests for mean residual life." Biometrika, 62, 585-593.

[6] Meilijson, I. (1972). "Limiting properties of the mean residual lifetime function." Annals of Mathematical Statistics, 43 (1), 354-357.

[7] Muth, E. J. (1977). "Reliability models with positive memory derived from the mean residual life function," in Theory and Applications of Reliability, (C. P. Tsokos and I. N. Shimi eds.), Academic Press, 401-434.

[8] Oakes, D. and Dasu, T. (1990). "A note on residual life." Biometrika, 77, 409-410.

[9] Rojo, J. (1996). "Relationships between pure tail orderings of lifetime distributions and some concepts of residual life." Annals of the Institute of Statistical Mathematics, 48 (2), 247-255. 
[10] Snyder, C. (1982). "Kummer congruences for the coefficients of Hurwitz series." Acta Arithmetica, XL, 175-191. 